\title{
A value-at-risk approach to optimisation of warranty policy
}

\author{
Ming Luo, Shaomin $\mathrm{Wu}^{1}$ \\ Kent Business School, University of Kent, \\ Canterbury, Kent CT2 7FS, United Kingdom
}

\begin{abstract}
In the real world, a manufacturer may produce many products, which may have common components installed. Consequently, the frequencies of the warranty claims of those products are statistically dependent. Warranty policy optimisation in the existing research, however, has not considered such statistical dependence, which may increase bias in decision making. This paper is the first attempt to collectively optimises warranty policy for a set of different products, produced by one manufacturer, whose failures are statistically dependent, using tools borrowed from financial mathematics (i.e., value-at-risk theory and copula). We prove the existence of the optimal solutions for different scenarios. Numerical examples are used to validate the applicability of the proposed methods.
\end{abstract}

Keywords: $(\mathrm{T})$ value-at-risk, warranty policy optimisation, mean-risk, copulas.

\section{Introduction}

Warranty is essentially offered with most durable products, which may be legally mandated or market driven: the European Union (EU) passed legislation requiring a two-year warranty for all products sold in Europe (S. Wu, 2014b). Warranty expense is an important part of a manufacturer's operating expense, for example, the automotive industry is the most warrantyintensive of all: the total automotive OEM (original equipment manufacturer) warranty claims paid by the entire U.S.-based manufacturers is $\$ 10,097$ million in 2015 (WarrantyWeek, 2016). To improve a manufacturer's operating expense management, an efficient warranty policy management supported by warranty data analysis and modelling is necessary. It is known that the number of warranty claims and the associated cost are uncertain, and products with longer warranty periods may attract more buyers than those with shorter warranty periods. From a

$\overline{1}$ Corresponding author. Email: s.m.wu@kent.ac.uk. Telephone: 00441227827940. 
manufacturer's perspective, however, providing a longer warranty period implies more resources that are needed to handle warranty claims. Hence, there is a need to develop approaches to optimising the warranty price and the warranty length.

In the literature, many methods aiming to optimise the warranty price and the warranty length of an individual product have been proposed. Fig. 1 illustrates the evolution of the research in warranty policy optimisation, which shows that the research evolves from simple and unrealistic assumptions to more complex and realistic ones.

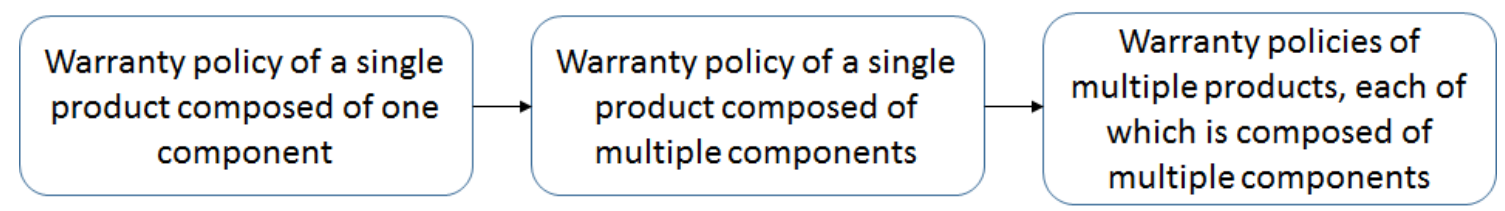

Fig. 1. Evolution of warranty policy optimisation

At the early stage, many researchers attempt to find the optimal price and warranty length, assuming that the product is composed of only one component (see the most-left rectangle in Fig. 1). At the same time, some other factors, such as production rate, market competition and demand, etc., are also considered. Ladany and Shore (2007) address a method to determine the optimal warranty period with considering the products lifetime and market demand. Lin, Wang, and Chin (2009) optimise the price, warranty length and production rate of a one component system dynamically. Wu, Chou, and Huang (2009) develop a decision model to determine the optimal price, the length of warranty and the production rate to maximise profit based on the pre-determined life cycle in a static demand market. Aggrawal, Anand, Singh, and Singh (2014) present a method to optimise warranty price and the length of warranty for a product based on a two-dimensional innovation diffusion model, and estimate the overall maximum profit for the manufacturer. Wei, Zhao, and Li (2015) investigates the optimal strategies on product price and the length of warranty of two products produced by two manufacturers and sold by one dealer. Yazdian, Shahanaghi, and Makui (2016) jointly optimises the acquisition price, re-manufacturing degree, selling price and the length of warranty of re-manufacturing products under linear and non-linear demand functions. Lei, Liu, and Shum (2017) price warranty policy of a single product dynamically with considering consumer learning, i.e. consumers' demand reacting on the warranty price change; and find warranty sales do not generate profit directly though are profitable overall.

The one-component assumption of a single product may be too unrealistic. Researchers then consider the assumption that a product is composed of multiple components (see the middle rectangle in Fig. 1). Huang, Liu, and Murthy (2007) develop a model to determine the optimal product reliability, price and warranty strategy to achieve the maximum total integrated profit for a general repairable multi-component product sold under a free replacement-repair warranty strategy. Matis, Jayaraman, and Rangan (2008) explore the optimal price and pro rate warranty length for a multi-component product with considering the different repair options on the com- 
ponents. Bai and Pham (2006) investigate optimisation of warranty policies for single products composed of multiple components. Liu, Wu, and Xie (2015) also investigate the warranty cost for a single product consisting of multiple components; meanwhile, as an improvement, the failure interactions between the components are considered. Ahmadi (2016) addresses an optimal replacement problem for complex multi-component systems by determining an optimal operating time which balances income and cost to maximizes the expected profit over a cycle. Adkins and Paxson (2017) construct a general replacement model for a multi-component product with considering the salvage value and depreciation in operating. Chen, Lo, and Weng (2017) seek to maximize the total profit per item of a multi-component product through optimally determine the production run length and the warranty period.

All of the literature mentioned above solely maximises the profit of individual products produced by a manufacturer. Little research, however, has been devoted to optimizing warranty policy of a set of products collectively. However, in real world, a manufacturer may assemble different types of product by sharing some key components; then, the manufacturer has to deal with the warranty policies for multiple products considered as multi-component systems, which is the third stage of warranty policy optimisation research (see the most-right rectangle in Fig. 1) and investigated in this paper.

The warranty claim arrival processes of products may not be statistically independent because the claims may have common causes such as similar design, same production lines and same types of components installed in the products. For example, Apple, iPhone 6, iPhone 6 Plus and iPad Mini 4, have the same type of CPU. If any design or quality problems happen on one of the products, warranty claims of the other products will crop up during a short period.

As can be seen from the above literature review, however, little attention has been paid to collectively optimise warranty policies for a portfolio of different products. This motivates us to develop novel approaches to filling in the knowledge gap.

We propose to optimise warranty policy through maximising product profit. The method collectively optimises the warranty price and the warranty length of a set of different products whose warranty claims are statistically dependent, considering the uncertainty of the product profit. The value-at-risk theory is borrowed to manage the uncertainty. The dependence is modelled by copulas, a tool from the probability theory. The use of copulas provides a more flexible tool to model more complicated dependence than a simple method such as covariance estimation.

The novelty of this paper lies in the fact that it is the first attempt to collectively optimise the warranty policies of a portfolio of products from a manufacturer's perspective.

The rest sections of the paper are structured as follows. Section 2 lists the assumptions of the optimisation problems. It also formulates the profits and warranty costs of individual products and portfolio of products, respectively. Section 3 investigates the existence of the optimal solutions for the different optimisation problems and uses copulas to model the dependence among warranty claims of different products. Section 4 offers numerical examples to illustrate 
the proposed methods and to validate the applicability of the proposed methods. Section 5 concludes the paper and proposes our future work.

\section{Formulation of the problem}

Assume a manufacturer offers non-renewing free replacement warranty (NFRW) policies. Under an NFRW policy, the manufacturer provides its customers with repair or replacement at no cost within the warranty period; the original warranty is not altered upon a failed item; and the manufacturer only guarantees satisfactory service on the item within the original warranty period. Assume that repair time is negligible and the repair is minimal repair. Products are new at $t=0$ when they are sold. The number of claims follows the non-homogeneous Poisson process (NHPP). The numbers of warranty claims and the claim cost are statistically independent.

The notations in Table 1 are used throughout this paper.

Table 1

Notation table

$X_{k, i} \quad$ Cost of the $i$ th warranty claim of product $k$

$N_{k}(t)$ Number of warranty claims of product $k$ within time interval $(0, t)$

$S_{k}(t)$ Total cost of warranty claims of product $k$ within time interval $(0, t)$

$P_{k} \quad$ Price of product $k$

$T_{k} \quad$ Warranty length of product $k$

$\boldsymbol{P}$ Vector of prices of products

$\boldsymbol{T}$ Vector of lengths of warranties

$\lambda_{k}$ Parameter of the claim arrival process of product $k$

$\mu_{k} \quad$ Expected cost per claim of product $k$, mean of $X_{k, i}$

$\sigma_{k} \quad$ Standard deviation of $X_{k, i}$

$M_{k} \quad$ Sales volume of product $k$, which is a function of $P_{k}$ and $T_{k}$

$M$ Vector of all sales volume

$\omega_{k}$ Profit of product $k$, which is a function of $P_{k}$ and $T_{k}$

$\Omega$ Total profit of the manufacturer's products portfolio

\subsection{The sales volume and profit}

For a product, there are two critical marketing variables: the selling price and the warranty length (Chen et al., 2017). The sales volume of a product is negatively related to its selling 
price and positively related to its warranty length. Denote $P_{k}$ and $T_{k}$ as the selling price $P_{k}$ and the warranty length $T_{k}$ of product $k$, respectively. Both $P_{k}$ and $T_{k}$ can influence the sales volume, $M_{k}$, and profit, $\omega_{k}$. In what follows, the profit of product $k$ is the revenue deducting the warranty cost, i.e. $\omega_{k}=M_{k} P_{k}-S_{k}\left(T_{k}\right)$, where $S_{k}\left(T_{k}\right)$ is the aggregated warranty cost of product $k$ within $T_{k}$.

In the literature, the sales volume of product $k, M_{k}$, is expressed by a function of product price $P_{k}$ and length of warranty $T_{k}$ in different forms, including linear (Lin et al., 2009; Yazdian et al., 2016) and non-linear ones (Huang et al., 2007; Ladany \& Shore, 2007; Xie, Liao, \& Zhu, 2014). For simplicity, a linearity form, introduced by Yazdian et al. (2016), is used in this paper. The sales volume is defined by

$$
M_{k}=A_{k}-\beta_{k} P_{k}+\eta_{k} T_{k}
$$

where $A_{k}(>0)$ is a constant relating to the market size of product $k$, and $\beta_{k}(>0)$ and $\eta_{k}(>0)$ are the price and length of warranty elasticities, respectively.

\subsection{The distribution of the aggregated warranty cost}

Suppose a manufacturer produces $n$ products. The aggregated warranty cost of product $k$ follows a stochastic process $\left\{S_{k}\left(T_{k}\right)\right\}_{T_{k} \geq 0}$ over the warranty period $\left(0, T_{k}\right)$, which is expressed by the following equation,

$$
S_{k}\left(T_{k}\right)=\sum_{j=1}^{N_{k}\left(T_{k}\right)} X_{k, j},
$$

where $X_{k, j}$ is the cost of the $j$-th claim of product $k$ and $N_{k}\left(T_{k}\right)$ is the number of claims during $\left(0, T_{k}\right) .\left\{X_{k, 1}, X_{k, 2}, \ldots, X_{k, j}\right\}$ are independent and identically distributed random variables which have finite values on the positive half-line $\mathbb{R}_{>0}$ with the probabilities $P\left(X_{k, j}\right)$. The cost of claims is assumed to follow the log-normal distribution. The counting process $N_{k}\left(T_{k}\right)$ is assumed to take a form of the NHPP with cumulative intensity $M_{k} \Lambda_{k}\left(T_{k}\right)$, and $P\left(N_{k}\left(T_{k}\right)=n\right)=\frac{\left(M_{k} \Lambda_{k}\left(T_{k}\right)\right)^{n}}{n !} e^{-M_{k} \Lambda_{k}\left(T_{k}\right)} . N_{k}(t)$ and $X_{k, i}$, are assumed to be statistically independent.

The expected value of $S_{k}\left(T_{k}\right)$ is given by

$$
\mathrm{E}\left[S_{k}\left(T_{k}\right)\right]=\mathrm{E}\left[N_{k}\left(T_{k}\right)\right] \mathrm{E}\left[X_{k}\right]=M_{k} \Lambda_{k}\left(T_{k}\right) \mu_{k}
$$

and the variance of $S_{k}\left(T_{k}\right)$ is given by

$$
\begin{aligned}
\operatorname{Var}\left[S_{k}\left(T_{k}\right)\right] & =\mathrm{E}\left[N_{k}\left(T_{k}\right)\right] \operatorname{Var}\left[X_{k}\right]+\operatorname{Var}\left[N_{k}\left(T_{k}\right)\right] \mathrm{E}\left[X_{k}\right]^{2} \\
& =M_{k} \Lambda_{k}\left(T_{k}\right)\left(\operatorname{Var}\left[X_{k}\right]+\mathrm{E}\left[X_{k}\right]^{2}\right) \\
& =M_{k} \Lambda_{k}\left(T_{k}\right)\left(\sigma_{k}^{2}+\mu_{k}^{2}\right)
\end{aligned}
$$


where $\mu_{k}$ and $\sigma_{k}$ are the mean and the variance of $X_{k}$, respectively.

Denote $F_{S_{k}}$ and $f_{S_{k}}$ as the probability density function (pdf) and the cumulative distribution function (cdf) of $S_{k}\left(T_{k}\right)$, respectively. The characteristic function of $X_{k}$ is $\varphi_{X_{k}}(t)=$ $\int_{-\infty}^{\infty} f_{X_{k}}(x) e^{i t x} d x$, where $i$ is a unit imaginary number. Denote $\varphi_{S_{k}}(t)$ as the characteristic function of $S_{k}$. The probability generation function of $N_{k}\left(T_{k}\right)$ is $\psi_{k}(s)=\sum_{n=0}^{\infty} s^{n} p_{k, n}$, where $p_{k, n}=\operatorname{Pr}\left\{N\left(T_{k}\right)=n\right\}$. According to the Levy-Khintchine formula, one can obtain

$$
\varphi_{S_{k}}(t)=\sum_{n=0}^{\infty}\left(\varphi_{X_{k}}(t)\right)^{n} p_{k, n}=e^{M_{k} \Lambda_{k}\left(T_{k}\right)\left(\varphi_{X_{k}}(t)-1\right)}
$$

Then, the density of $S_{k}\left(T_{k}\right)$ can be calculated through the inverse Fourier transform, that is

$$
f_{S_{k}}(x)=\frac{1}{2 \pi} \int_{-\infty}^{\infty} \varphi_{S_{k}}(t) e^{-i t x} d t=\frac{1}{2 \pi} \int_{-\infty}^{\infty} e^{M_{k} \Lambda_{k}\left(T_{k}\right)\left(\varphi_{X_{k}}(t)-1\right)-i t x} d t .
$$

Apparently, $S_{k}\left(T_{k}\right)$ is non-negative. According to Luo and Shevchenko (2009), the probability density and cumulative distribution function of $S_{k}\left(T_{k}\right)$ are given by

$$
f_{S_{k}}(z)=\frac{2}{\pi} \int_{0}^{\infty} R e\left[\varphi_{S_{k}}(t)\right] \cos (t z) d t
$$

and

$$
F_{S_{k}}(z)=\frac{2}{\pi} \int_{0}^{\infty} \operatorname{Re}\left[\varphi_{S_{k}}(t)\right] \frac{\sin (t z)}{t} d t
$$

respectively, where $z \geq 0$.

In practice, computing the density and cumulative distribution through the Fourier transform requires high computing power to deal with the underflow and overflow problems. Another popular approach is to use an approximating distribution to avoid direct calculation of Eq. (7) and Eq. (6). According to Bee (2016), if the distribution of $X_{k}$ is subexponential, the compound $S_{k}\left(T_{k}\right)$ inherits the subexponentiality property from $X_{k}$. In this case, $X_{k}$ is assumed to follow the log-normal distribution, which is subexponential. Hence, $S_{k}\left(T_{k}\right)$ is also subexponential, and $X_{k}$ and $S_{k}\left(T_{k}\right)$ are tail equivalent. For details about the definition of tail equivalent, the reader is referred to Bee (2016).

To make this paper concise, we use the log-normal distribution to approximate $S_{k}\left(T_{k}\right)$.

\subsection{Product profit}

In this subsection, we derive the profit of products for different scenarios.

\subsubsection{The profit of one product}

The profit of product $k$ is given by

$$
\omega_{k}=M_{k} P_{k}-S_{k}\left(T_{k}\right) .
$$


Denote $F_{\omega_{k}}$ and $f_{\omega_{k}}$ as the cdf and pdf of $\omega_{k}$, respectively. Then

$$
\begin{aligned}
F_{\omega_{k}}(z) & =P\left[\omega_{k} \leq z\right] \\
& =P\left[M_{k} P_{k}-S_{k}\left(T_{k}\right) \leq z\right] \\
& =P\left[S_{k}\left(T_{k}\right) \geq M_{k} P_{k}-z\right] \\
& =1-F_{S_{k}}\left(M_{k} P_{k}-z\right),
\end{aligned}
$$

which can be calculated on the basis of Eq. (7).

The expected value of $\omega_{k}$ is

$$
\mathrm{E}\left[\omega_{k}\right]=M_{k}\left[P_{k}-\Lambda_{k}\left(T_{k}\right) \mu_{k}\right]
$$

and the variance of $\omega_{k}$ is

$$
\operatorname{Var}\left[\omega_{k}\right]=\operatorname{Var}\left[S_{k}(t)\right]=M_{k} \Lambda_{k}\left(T_{k}\right)\left(\sigma_{k}^{2}+\mu_{k}^{2}\right)
$$

\subsubsection{The profit of a portfolio of products}

The total profit of a portfolio consisting of $N$ products is given by

$$
\Omega=\sum_{k=1}^{N} \omega_{k}=\sum_{k=1}^{N}\left[M_{k} P_{k}-S_{k}\left(T_{k}\right)\right] .
$$

The distribution of the profit of product portfolio can be expressed by

$$
F_{\Omega}(z)=P\{\Omega \leq z\}=1-F^{(N)}(z)
$$

where $F^{(N)}(z)$ is the $N$-fold convolution of the distribution of $\omega_{k}$. The expected total profit is

$$
\mathrm{E}[\Omega]=\sum_{k=1}^{N} \mathrm{E}\left[\omega_{k}\right]=\sum_{k=1}^{N} M_{k}\left[P_{k}-\Lambda_{k}\left(T_{k}\right) \mu_{k}\right]
$$

As we mentioned in Section 1.2, the warranty claim arrival processes of the products produced by the same manufacturer may be correlated. Hence the variance of the portfolio profit is related to the correlation among the products. If the claim arrival processes of different products are linearly correlated, the variance of $\Omega$ can be calculated based on the covariance matrix of the portfolio, which is

$$
\operatorname{Var}[\Omega]=\boldsymbol{I}^{T} \boldsymbol{V} \boldsymbol{I}
$$


where $\boldsymbol{I}^{T}=[1,1, \ldots, 1]$ and

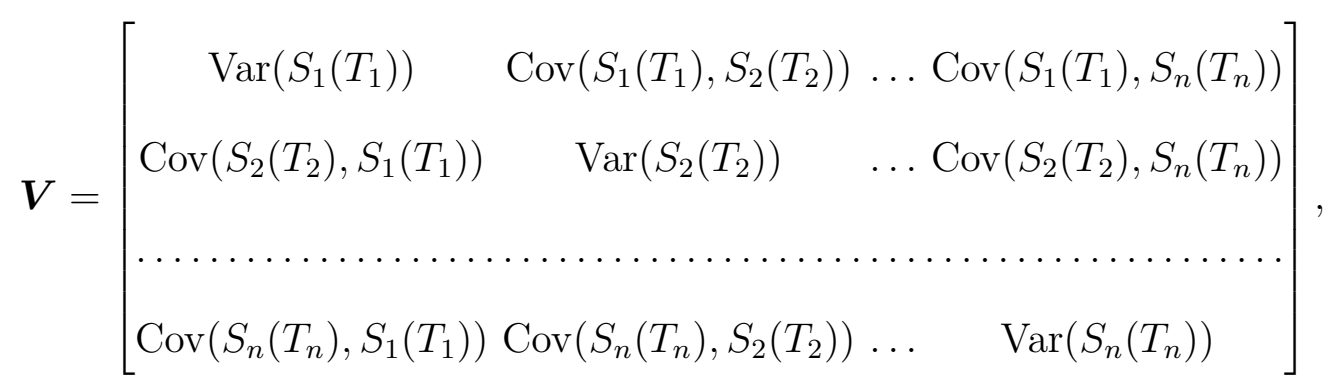

where $\operatorname{Cov}\left(S_{k}\left(T_{k}\right), S_{i}\left(T_{i}\right)\right)=\rho_{k, i} \sqrt{\operatorname{Var}\left(S_{k}\left(T_{k}\right)\right) \operatorname{Var}\left(S_{i}\left(T_{i}\right)\right)}$, and $\rho_{k, i}$ is the Pearson correlation coefficient. The warranty costs of different products may have different types of dependence such as a rank correlation or a tail-dependence. In such cases, the metrics that can only measure a linear correlation may be inappropriate in the case where the relationship of the variables is not linear. As such, we employ a powerful tool, copula, to model the dependence among the products, which will be discussed in Section 3.3. According to Boubaker and Sghaier (2013), Kendall's tau and copula parameters, especially the parameters of the Archimedean copulas, can be used as substitutes (and more comprehensive metrics) for measuring both the linear and the nonlinear relationships in the covariance matrix in portfolio optimisation.

\section{Mean-risk optimisation}

In this paper, the mean-risk optimisation is used to maximise the expected profit under an acceptable risk level. The objective functions are illustrated in Eq. (10) and Eq. (14) for one product and multiple products scenarios, respectively. The constraints of optimisation problems are given for different risk measures.

In the one product scenario, the manufacturer aims to maximise the following function:

$$
\mathrm{E}\left[\omega_{k}\right]=M_{k}\left[P_{k}-\Lambda_{k}\left(T_{k}\right) \mu_{k}\right]
$$

Suppose in this case, the NHPP following the cumulative intensity $\Lambda_{k}\left(T_{k}\right)=a_{k} T_{k}^{b_{k}}$ (where $\left.b_{k}>1\right)$ are used. Then, the objective function is defined by

$$
\mathrm{E}\left[\omega_{k}\right]=A_{k} P_{k}-A_{k} \mu_{k} a_{k} T_{k}^{b_{k}}-\beta_{k} P_{k}^{2}+\beta_{k} \mu_{k} P_{k} a_{k} T_{k}^{b_{k}}+\eta_{k} P_{k} T_{k}-\eta_{k} \mu_{k} a_{k} T_{k}^{b_{k}+1}
$$

In case both of $P_{k}$ and $T_{k}$ are decision variables, based on the properties of this function, we have Proposition 1.

Proposition 1 Depending on whether $P_{k}$ and $T_{k}$ are known, one can prove the following results.

- If both of $P_{k}$ and $T_{k}$ are decision variables, the global maxima of $\mathrm{E}\left[\omega_{k}\right]$ does not exist.

- If $T_{k}\left(\right.$ or $\left.P_{k}\right)$ is the decision variable and $P_{k}$ (or $T_{k}$ ) is known, the global maxima of the 
function $\mathrm{E}\left[\omega_{k}\right]\left(T_{k}\right)$ (or $\mathrm{E}\left[\omega_{k}\right]\left(P_{k}\right)$ ) exists.

The proof of Proposition 1 is presented in the Appendix.

In a multiple product scenario, the objective function to be maximised is

$$
\mathrm{E}[\Omega]=\sum_{k=1}^{N} M_{k}\left(P_{k}-\Lambda_{k}\left(T_{k}\right) \mu_{k}\right) .
$$

Since the prices and sales volumes are assumed to be mutually independent in this paper, Proposition 1 is also valid in the multiple products scenario. The dependence among the warranty claims of the products is reflected in the constraints of optimisations.

\subsection{The risk measure}

In finance, Artzner, Delbaen, Eber, and Heath (1999) define risk as the variability of the future value of a position due to uncertain events. Babaei, Sepehri, and Babaei (2015) point out that risk is used to characterise the situation in which a portfolio is exposed to vulnerabilities and enforces losses to the institutions. Risk measures are introduced for the requirement of quantifying the losses that may be incurred. The variance of a random variable is considered as a risk measure by the overwhelming influential models of portfolio selection. However, since the variance is a symmetric risk measure, researchers turn to using downside risk measures, such as Value-at-Risk (VaR) and Conditional VaR (Expected Shortfall), which can reflect a better notion of risk (Babaei et al., 2015). Furthermore, the variance as a risk measure is normally applied under the assumption that the correlation is linear. Such an assumption is not imposed in the VaR and CVaR theories. In this paper, we focus on maximising the profit under the mean-risk framework, in which the risk may be measured by the variance, VaR or CVaR.

\subsection{One product scenario}

In the one product scenario, the manufacturer aims to maximise the expected profit of one product at an acceptable risk level. The optimisation problems with different risk measures are discussed in the following subsections.

\subsubsection{Mean-variance framework}

A mean-variance framework aims to maximise the expected profit of product $k$ under a given/acceptable value of variance. The optimisation problem is defined by

$$
\begin{gathered}
\max \quad \mathrm{E}\left[\omega_{k}\right]=M_{k}\left[P_{k}-\Lambda_{k}\left(T_{k}\right) \mu_{k}\right], \\
\text { s.t. } \quad \operatorname{Var}\left[\omega_{k}\right]=M_{k} \Lambda_{k}\left(T_{k}\right)\left(\sigma_{k}^{2}+\mu_{k}^{2}\right) \leq \psi, \\
M_{k}=A_{k}-\beta_{k} P_{k}+\eta_{k} T_{k} \geq 0,
\end{gathered}
$$




$$
\begin{gathered}
P_{k}>0, \\
T_{k} \geq L_{T},
\end{gathered}
$$

where $\psi$ is the acceptable risk level of the manufacturer, and $L_{T}$ is the legal minimum limit of the length of warranty, e.g. the manufacturers should provide at least 2-year warranty in Europe. According to Proposition 1, only one variable, either $P_{k}$ or $T_{k}$, is treated as the decision variable in optimisation. Regarding this mean-variance optimisation, for example, if $P_{k}$ is the decision variable, the constraint is $P_{k} \geq \frac{1}{\beta_{k}}\left(A_{k}+\eta_{k} T_{k}-\frac{\psi}{a_{k} T_{k}^{b_{k}}\left(\sigma_{k}^{2}+\mu_{k}^{2}\right)}\right)$, and the objective function can achieve the global maxima at $P_{k}=\frac{A_{k}+\beta_{k} \mu_{k} a_{k} T_{k}^{b_{k}}+\eta_{k} T_{k}}{2 \beta_{k}}$. If $\frac{1}{\beta_{k}}\left(A_{k}+\eta_{k} T_{k}-\frac{\psi}{a_{k} T_{k}^{b k}\left(\sigma_{k}^{2}+\mu_{k}^{2}\right)}\right)<$ $\frac{A_{k}+\beta_{k} \mu_{k} a_{k} T_{k}^{b_{k}}+\eta_{k} T_{k}}{2 \beta_{k}}$, the objective function is maximized at $P_{k}=\frac{A_{k}+\beta_{k} \mu_{k} a_{k} T_{k}^{b_{k}}+\eta_{k} T_{k}}{2 \beta_{k}}$, otherwise, the objective function is maximized at $P_{k}=\frac{1}{\beta_{k}}\left(A_{k}+\eta_{k} T_{k}-\frac{\psi}{a_{k} T_{k}^{b_{k}}\left(\sigma_{k}^{2}+\mu_{k}^{2}\right)}\right)$. Then, we have Proposition 2.

Proposition 2 If $T_{k}$ (or $P_{k}$ ) is the decision variable, $P_{k}$ (or $T_{k}$ ) is known and the power law parameter $b_{k}>1$, then the optimal solution, which maximises the expected profit of product $k$ under a given variance level, exists.

The proof of Proposition 2 can be find in the Appendix.

\subsubsection{Mean-VaR framework}

In general, value-at-risk is the $\alpha$-quantile of a distribution, where $\alpha$ is a given confidence level. In this case, $\omega_{k}$ is the profit of product $k$, we defined the $\operatorname{VaR}_{\alpha}\left(\omega_{k}\right)$ as the minimum profit at the $(1-\alpha)$ level, i.e. there is a $(1-\alpha)$ probability that the profit of product $k$ will be greater than $\operatorname{VaR}_{\alpha}\left(\omega_{k}\right)$. The $\operatorname{VaR}_{\alpha}\left(\omega_{k}\right)$ can be expressed as

$$
\operatorname{VaR}_{\alpha}\left(\omega_{k}\right)=F_{\omega_{k}}^{-1}(\alpha)
$$

According to Eq. (9),

$$
\operatorname{VaR}_{\alpha}\left(\omega_{k}\right)=M_{k} P_{k}-F_{S_{k}}^{-1}(1-\alpha) .
$$

Then, the optimisation problem can be defined by

$$
\begin{gathered}
\max \quad \mathrm{E}\left[\omega_{k}\right]=M_{k}\left(P_{k}-\Lambda_{k}\left(T_{k}\right) \mu_{k}\right), \\
\text { s.t. } \quad \operatorname{VaR}_{\alpha}\left(\omega_{k}\right)=M_{k} P_{k}-F_{S_{k}}^{-1}(1-\alpha) \geq \phi, \\
M_{k} \geq 0, \quad P_{k}>0, \quad T_{k} \geq L_{T},
\end{gathered}
$$

where $\alpha$ is a given confidence level, and $\phi$ is the minimum profit at the $(1-\alpha)$ confidence level set by the manufacturer. If $\phi<0$, the maximum loss should be less than $-\phi$ at the $(1-\alpha)$ confidence level. If $P_{k}$ is the decision variable and $T_{k}$ is known, then $M_{k} P_{k}$ in $\operatorname{VaR}_{\alpha}\left(\omega_{k}\right)=$ $M_{k} P_{k}-F_{S_{k}}^{-1}(1-\alpha) \geq \phi$ is a parabola with a negative coefficient on the quadratic term, and $F_{S_{k}}^{-1}(1-\alpha)$ can be derived from the distribution of warranty cost $S_{k}$. If $T_{k}$ is known, the mean 
and the variance of $S_{k}$ are $\left(A_{k}-\beta_{k} P_{k}+\eta_{k} T_{k}\right) \Lambda_{k}\left(T_{k}\right) \mu_{k}$ and $\left(A_{k}-\beta_{k} P_{k}+\eta_{k} T_{k}\right) \Lambda_{k}\left(T_{k}\right)\left(\sigma_{k}^{2}+\mu_{k}^{2}\right)$, respectively. Both of the quantities monotonously decrease with respect of $P_{k}$. $F_{S_{k}}^{-1}(1-\alpha)$ decreases monotonously with $P_{k}$ as well. As such, the feasible range of $P_{k}$ is $\left(\max \left(0, P_{l}\right), P_{u}\right)$, where $P_{l}$ and $P_{u}$ are defined by $\operatorname{VaR}_{\alpha}\left(\omega_{k}\right)=M_{k} P_{k}-F_{S_{k}}^{-1}(1-\alpha) \geq \phi$, respectively.

\subsubsection{Mean-CVaR framework}

Denote $\operatorname{CVaR}_{\alpha}\left(\omega_{k}\right)$ as the conditional value-at-risk, where $\alpha$ is a given probability. if the profit exceeds the $\operatorname{VaR}_{\alpha}\left(\omega_{k}\right)$ on the left tail, $\operatorname{CVaR}_{\alpha}\left(\omega_{k}\right)$ is the expected profit of the product $k$. In other word, $\operatorname{CVaR}_{\alpha}\left(\omega_{k}\right)$ is the expected profit of the product $k$ in the worst $\alpha \%$ of cases. In this paper, $\operatorname{CVaR}_{\alpha}\left(\omega_{k}\right)$ is expressed as

$$
\operatorname{CVaR}_{\alpha}\left(\omega_{k}\right)=\frac{1}{\alpha} \int_{-\infty}^{\operatorname{VaR}_{\alpha}\left(\omega_{k}\right)} z f_{\omega_{k}}(z) d z
$$

where $f_{\omega_{k}}(z)$ is the pdf of $\omega_{k}$. The distribution of $\omega_{k}$ is determined by the distribution of $S_{k}$. Hence $\mathrm{CVaR}_{\alpha}\left(\omega_{k}\right)$ can be calculated by

$$
\mathrm{CVaR}_{\alpha}\left(\omega_{k}\right)=M_{k} P_{k}-\frac{1}{\alpha} \int_{F_{S_{k}}^{-1}(1-\alpha)}^{+\infty} z f_{S_{k}}(z) d z
$$

where $f_{S_{k}}(z)$ and $F_{S_{k}}^{-1}(1-\alpha)$ are defined by Eq. (6) and Eq. (7), respectively. They can be approximated by the log-normal distribution, as shown in Section 4. The optimisation problem can therefore be expressed by

$$
\begin{gathered}
\max \mathrm{E}\left[\omega_{k}\right]=M_{k}\left(P_{k}-\Lambda_{k}\left(T_{k}\right) \mu_{k}\right), \\
\text { s.t. } \mathrm{CVaR}_{\alpha}\left(\omega_{k}\right)=M_{k} P_{k}-\frac{1}{\alpha} \int_{F_{S_{k}}^{-1}(1-\alpha)}^{+\infty} z f_{S_{k}}(z) d z \geq \delta, \\
M_{k} \geq 0, P_{k}>0, T_{k} \geq L T,
\end{gathered}
$$

where $\alpha$ is a given confidence level and $\delta$ is the acceptable minimum level of $\mathrm{CVaR}_{\alpha}\left(\omega_{k}\right)$ set by the manufacturer.

Referring to Propositions 1 and 2, and the above discussions in Section 3.2.2 and 3.2.3, one can obtain the following proposition.

Proposition 3 For $T_{k}$ and $P_{k}$, if one of them is the decision variable and the other is known, then the optimal solution, which maximises the expected profit of product $k$ under a given valueat-risk level or conditional value-at-risk level, exists.

\subsection{Multiple product scenario}

The preceding section investigates the scenarios of the optimisation problems of individual products under a risk-informed consideration.

Assuming that a manufacturer produces $N$ products, one can easily estimate the expected 
total profit through estimating and then summing the expected profit of each individual product. Considering the uncertainty in estimation of the total profit of the $N$ products, one may consider the statistical dependence among the warranty claim arrival processes. In this section, the portfolio optimisation is investigated under the mean-variance, mean-VaR and mean-CVaR frameworks.

\subsubsection{Mean-Variance framework}

Under the mean-variance framework for the $N$ products, the optimisation problem is defined by

$$
\max \quad \mathrm{E}[\Omega]=\sum_{k=1}^{N} M_{k}\left(P_{k}-\Lambda_{k}\left(T_{k}\right) \mu_{k}\right)
$$

$$
\text { s.t. } \operatorname{Var}[\Omega]=\sum_{k=1}^{N} M_{k} T_{k} \lambda_{k}\left(\sigma_{k}^{2}+\mu_{k}^{2}\right)+2 \sum_{i \neq j}^{N} \rho_{i, j} \sqrt{M_{i} M_{j} T_{i} T_{j} \lambda_{i} \lambda_{j}\left(\sigma_{i}^{2}+\mu_{i}^{2}\right)\left(\sigma_{j}^{2}+\mu_{j}^{2}\right)} \leq \psi \text {. }
$$

In this optimisation problem, the dependence among the warranty claims of the different products is measured with the Pearson correlation coefficient $\rho_{i, j}$, and the objective function is the sum of the expected profits of the $N$ products. According to Proposition 2, one can obtain the following proposition.

Proposition 4 For $\boldsymbol{T}$ and $\boldsymbol{P}$, if one of them is the decision variable vector and the other is known, then the optimal solution, which maximises the expected total profit $\mathrm{E}[\Omega]$ of the product portfolio under a given variance level, exists.

\subsubsection{Mean-VaR framework}

Under the mean-VaR framework for the $N$ products, the optimisation problem is defined by

$$
\begin{gathered}
\max \mathrm{E}[\Omega]=\sum_{k=1}^{N} M_{k}\left(P_{k}-\Lambda_{k}\left(T_{k}\right) \mu_{k}\right), \\
\text { s.t. } \quad \operatorname{VaR}_{\alpha}[\Omega] \geq \phi .
\end{gathered}
$$

In practice, one may need to obtain the optimal solution. According to Babaei et al. (2015), even though the definition of the VaR is intuitive and easy to interpret, calculating the VaR of a portfolio is not easy. In financial mathematics, to calculate the portfolio VaR, there are three commonly used methods, which are the variance-covariance, stochastic simulation, and historical simulation methods. The variance-covariance method assumes that the risk factors are jointly normally distributed. The normality assumption may not hold in our case and therefore the variance-covariance method is not applicable. The VaR of the total profit of the product portfolio will be calculated using the stochastic simulation method in this research. The historical simulation method will be investigated in our future work. To conduct an exact and efficient simulation, copula, an important tool in the probability theory is borrowed.

Copulas are widely used in constructing multivariate distributions and formalising the dependence structures between random variables, whatever discrete or continuous. Abe Sklar 
first introduced the notion of copula in 1959 (Sklar, 1959). In recent years, copulas have attracted considerable attention in both theoretical and application aspects. Sklar's theorem states that any cumulative distribution function of a random vector can be written in terms of marginal distribution functions and a copula that describes the dependence structure between the variables (Sklar, 1959). Assume $\left(X_{1}, \ldots, X_{d}\right)$ is a given vector of random variables, its cumulative distribution function is $\left.H\left(x_{1}, \ldots, x_{d}\right)=P\left(X_{1} \leq x_{1}, \ldots, X_{d} \leq x_{d}\right)\right)$, and its marginals are $F_{k}\left(x_{k}\right)=P\left(X_{k} \leq x_{k}\right)$, where $k=1, \ldots d$. Sklar proved that $H\left(x_{1}, \ldots, x_{d}\right)=C\left(F_{1}\left(x_{1}\right), \ldots, F_{d}\left(x_{d}\right)\right)$, where $C($.$) is a copula. Copulas are useful in statistical applications because they allow one$ to estimate the marginals and the copula separately when modelling and estimating the distribution of a random vector. It has recently been used in modelling warranty claims $(\mathrm{S}$. Wu, 2014a).

Denote the joint distribution of the products' number of claims by

$$
H\left(z_{1}, z_{2}, \ldots, z_{k}\right)=C\left(F_{N_{1}}\left(z_{1}\right), F_{N_{2}}\left(z_{2}\right), \ldots, F_{N_{k}}\left(z_{k}\right)\right)
$$

where $C($.$) is a copula, and F_{N_{k}}$ is the CDF of the number of claims of product $k$. The density of the joint distribution is given by

$$
h\left(z_{1}, z_{2}, \ldots, z_{N}\right)=c\left(F_{N_{1}}\left(z_{1}\right), F_{N_{2}}\left(z_{2}\right), \ldots, F_{N_{k}}\left(z_{k}\right)\right) \prod_{k=1}^{n} f_{N_{k}}\left(z_{k}\right),
$$

where $c($.$) is the density of copula C($.$) .$

Then, the joint distribution of the products' profits can be simulated based on Eq. (6), Eq. (7), Eq. (22) and Eq. (23).

Let $F_{\Omega}(z)$ be the distribution of the total profit of the products, where $\Omega=\sum_{k=1}^{N} \omega_{k}$. In a copula-based model, the VaR of the total profit can be calculated through simulation. It is clear that calculating $F_{\Omega}(z)$ is mainly a numerical issue (Bernard \& Vanduffel, 2015).

\subsubsection{Mean-CVaR framework}

Under the mean-CVaR framework, the optimisation problem is defined by

$$
\begin{gathered}
\max \mathrm{E}[\Omega]=\sum_{k=1}^{N} M_{k}\left(P_{k}-\Lambda_{k}\left(T_{k}\right) \mu_{k}\right), \\
\text { s.t. } \quad \mathrm{CVaR}_{\alpha}[\Omega] \geq \delta .
\end{gathered}
$$

This optimisation problem implies that the manufacturer aims to maximise the total profit under the constraint that the expected extreme profit at confidence level $\alpha$ is not less than $\delta$. This constraint can also be expressed by the copula and marginal distributions.

Based on the property of the above objective function and Proposition 3, one can derive the following proposition

Proposition 5 For $\boldsymbol{T}$ and $\boldsymbol{P}$, if one of them is the decision variable vector and the other is 
known, then the optimal solution, which maximises the expected total profit $\mathrm{E}[\Omega]$ of the product portfolio under a given value-at-risk level or conditional value-at-risk level, exists.

\section{Numerical examples}

\subsection{One product scenario}

Assume that the cost of each warranty claim of one product, $X_{1}$, follows a log-normal distribution with mean $\mu_{1}=200$ and standard deviation $\sigma=40$; assume the warranty claim arrival process is a Non-homogeneous Poisson process with cumulative intensity $\Lambda_{1}\left(T_{1}\right)=0.004 T_{1}^{1.04}$; and the sales volume of this product is defined by $M_{1}=1,000-0.2 P_{1}+0.13 T_{1}$. In Table $2, L_{\mu_{1}}$ Table 2

Parameters for one product

\begin{tabular}{|l|l|l|l|}
\hline \multicolumn{4}{|l|}{ Log-normal } \\
\hline$\mu_{1}=200$ & $\sigma_{1}=40$ & $L_{\mu_{1}}=5.2787$ & $L_{\sigma_{1}}=0.1980$ \\
\hline NHPP & $b=1.04$ \\
\hline$a=0.004$ & \\
\hline \multicolumn{4}{|l|}{ Sales volume } \\
\hline$A_{1}=1,000$ & $\beta_{1}=0.2$ & $\eta_{1}=0.13$ & \\
\hline
\end{tabular}

and $L_{\sigma_{1}}$ are logarithmised mean and standard deviation respectively, and they are calculated by

$$
L_{\mu_{1}}=\ln \left(\frac{\mu_{1}}{\sqrt{1+\frac{\sigma_{1}^{2}}{\mu_{1}^{2}}}}\right)
$$

and

$$
L_{\sigma_{1}}=\sqrt{\ln \left(1+\frac{\sigma_{1}^{2}}{\mu_{1}^{2}}\right)} .
$$

\subsubsection{Mean-Variance}

Under the mean-variance framework, the optimisation problem is

$$
\begin{array}{cl} 
& \max \quad \mathrm{E}\left[\omega_{1}\right]=\left(1,000-0.2 P_{1}+0.13 T_{1}\right)\left(P_{1}-0.004 T_{1}^{1.04} \times 200\right) \\
\text { s.t. } & \operatorname{Var}\left[\omega_{1}\right]=\left(1,000-0.2 P_{1}+0.13 T_{1}\right) \times 0.004 T_{1}^{1.04} \times\left(200^{2}+40^{2}\right) \leq \psi .
\end{array}
$$

Suppose $T_{1}=720$. The above objective function is parabola and has the global maximum $\mathrm{E}\left[\omega_{1}\right]=1,113,257.59$ at $P_{1}=3,108.70$ with the corresponding sales volume $M_{1}=472$. Meanwhile, the feasible range of $P_{1}$ is $[5,468-0.000032 \psi, 5,468]$, which means: $P_{1}=3,108.70$ is the optimal solution if the variance limitation $\psi \geq 147,103,140.52$. 
If $P_{1}$ is known, for example, let $P_{1}=3,000$. The global maxima is $\mathrm{E}\left[\omega_{1}\right]=1,200,600$ when $T_{1} \approx 32$. The sales volume and the variance of total profit at $T_{1} \approx 32$ are $M_{1}=404$ and $\operatorname{Var}\left[\omega_{1}\right]=2,472,080.61$, respectively. Furthermore, the function of $\operatorname{Var}\left[\omega_{1}\right]$ monotonously increases with $T_{1}$. Hence, if the variance of the total profit is less than $\psi$ with $\psi \geq 2,472,080.61$, $T_{1}=32$ is the optimal solution. However, in practice, the length of warranty is regulated by the authorities, such as at least 2 years warranty is required in Europe, this regulation may also be considered in optimisation.

\subsubsection{Mean-VaR}

Under the mean-VaR framework, given that the confidence level $\alpha=0.05$, the optimisation problem is

$$
\begin{aligned}
& \max \quad \mathrm{E}\left[\omega_{1}\right]=\left(1,000-0.2 P_{1}+0.13 T_{1}\right)\left(P_{1}-200 \times 0.004 T_{1}^{1.04}\right) \\
& \text { s.t. } \quad \operatorname{VaR}_{0.05}\left(\omega_{1}\right)=\left(1,000-0.2 P_{1}+0.13 T_{1}\right) P_{1}-F_{S_{1}}^{-1}(0.95) \geq \phi .
\end{aligned}
$$

In this scenario, the effects of $P_{1}$ and $T_{1}$ on $\mathrm{VaR}_{0.05}$ should be investigated. However, the closed form of the cdf of $S_{1}$ is difficult to obtain, and we cannot obtain the close form of $F_{S_{1}}^{-1}(0.95)$ either. Then a log-normal distribution with mean, $\mathrm{E}\left[S_{1}\right]=0.8 T_{1}^{1.04} \times\left(1,000-0.2 P_{1}+0.13 T_{1}\right)$, and variance, $\operatorname{Var}\left[S_{1}\right]=166.4 T_{1}^{1.04} \times\left(1,000-0.2 P_{1}+0.13 T_{1}\right)$, is used to approximate the distribution $F_{S_{1}}(x)$.

Initially, we focus on the situation that $T_{1}$ is known. According to Section 4.1.1, if $T_{1}=720$, the objective function has a global maximum $\mathrm{E}\left[\omega_{1}\right]=1,113,257.59$ at $P_{1}=3,108.70$. then we have

$$
\operatorname{VaR}_{0.05}\left(\omega_{1}\right)=\left(1,093.6-0.2 P_{1}\right) P_{1}-F_{S_{1}}^{-1}(0.95) .
$$

If the constraint on $\operatorname{VaR}_{0.05}\left(\omega_{1}\right)$ is not considered, the feasible range of $P_{1}$ is $[0,5,468]$. Within this range, the relationship between $P_{1}$ and the first term in Eq. (24), i.e. the revenue (1,093.6$\left.0.2 P_{1}\right) P_{1}$, is illustrated in Fig. 2. The relationship between $P_{1}$ and the second term $F_{S_{1}}^{-1}(0.95)$ in Eq. (24), i.e. the right tail $5 \%$ VaR of warranty cost, is shown in Fig. 3. The relationship between $P_{1}$ and $\operatorname{VaR}_{0.05}\left(\omega_{1}\right)$ is illustrated in Fig. 4.

Fig. 3 reveals that the right tail $5 \%$ VaR of warranty cost monotonically decreases with $P_{1}$ within the feasible range of $P_{1}$. Then, the relationship between $\operatorname{VaR}_{0.05}\left(\omega_{1}\right)$ and $P_{1}$ is determined by the total revenue which is a quadratic function of $P_{1}$. Hence, with the optimal constraint, one can derive a feasible range of $P_{1},\left(p_{l 1}, p_{u 1}\right)$, where $p_{l 1}$ and $p_{u 1}$ are determined by $\phi$.

In accordance with the market behaviour, one may set market price $P_{1}$, said $P_{1}=3,000$. The optimal length of warranty $T_{1}$ can then be determined and one can obtain

$$
\operatorname{VaR}_{0.05}\left(\omega_{1}\right)=3,000 \times\left(400+0.13 T_{1}\right)-F_{S_{1}}^{-1}(0.95) .
$$

Fig. 5 and Fig. 6 reveal the effects of $T_{1}$ on the right tail $5 \%$ VaR of the warranty cost and $\operatorname{VaR}_{0.05}\left(\omega_{1}\right)$. The values of $T_{1}$ in these figures are discrete values from 2 years (720 days) to 10 years (3,600 days) with half of a year step (180 days). Then the optimal constraint gives a 


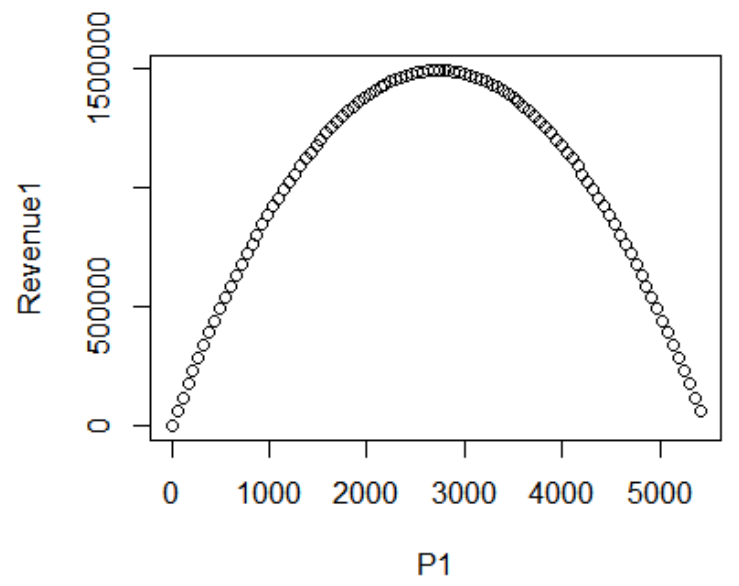

Fig. 2. Total revenue (on the $Y$-axis) against price $P_{1}$ (on the $X$-axis).

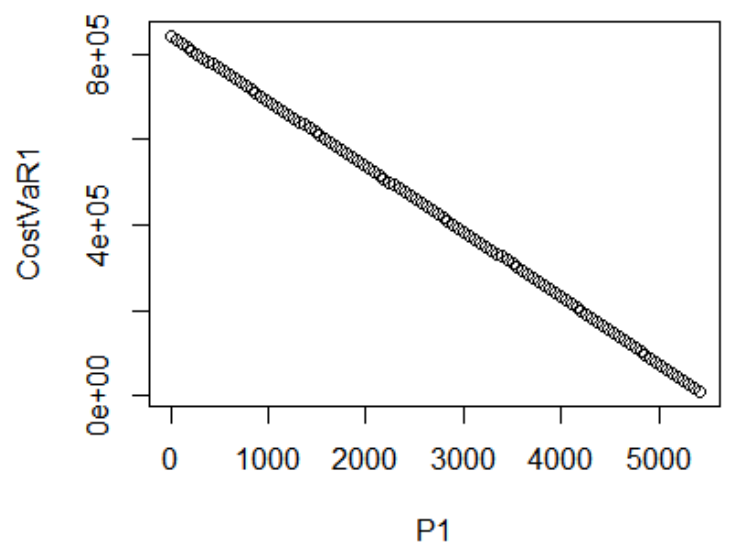

Fig. 3. Right tail $5 \%$ VaR of warranty cost (on the $Y$-axis) against $P_{1}$ (on the $X$-axis). feasible range of $T_{1},\left[720, t_{u}\right]$, where $t_{u}$ is determined by $\phi$.

\subsubsection{Mean-CVaR}

Under the mean-CVaR framework, the objective function is the same as that under the mean-VaR framework, but the constraint is given by

$$
\mathrm{CVaR}_{0.05}\left(\omega_{1}\right)=\left(1,000-0.2 P_{1}+0.13 T_{1}\right) P_{1}-\frac{1}{0.05} \int_{F_{S_{1}}^{-1}(0.95)}^{+\infty} z f_{S_{1}}(z) d z \geq \delta .
$$

The first term of Eq. (26) also is the total revenue of Product 1, and the second term is the right tail $5 \% \mathrm{CVaR}$ of the total warranty cost of Product $1 . f_{S_{1}}$ can also be approximated by the log-normal distribution.

If $T_{1}=720$ is known, the relationship between $P_{1}$ and $\mathrm{CVaR}_{0.05}\left(\omega_{1}\right)$ is illustrated in Fig. 7 . 


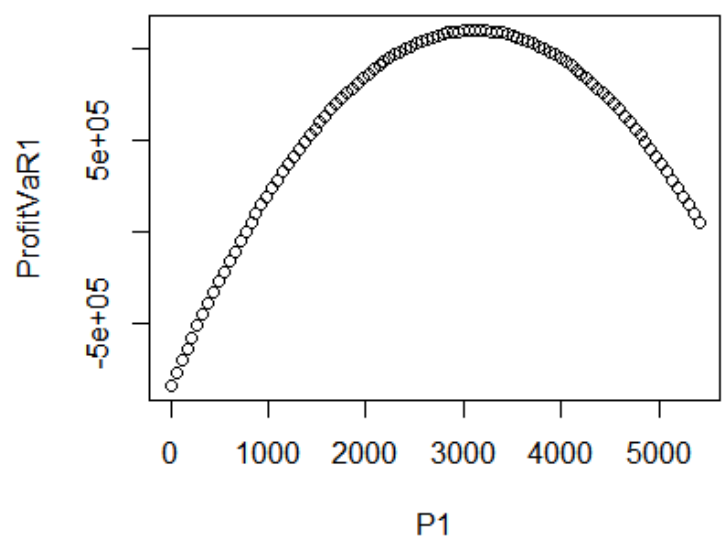

Fig. 4. $\operatorname{VaR}_{0.05}\left(\omega_{1}\right)$ (on the $Y$-axis) against $P_{1}$ (on the $X$-axis).

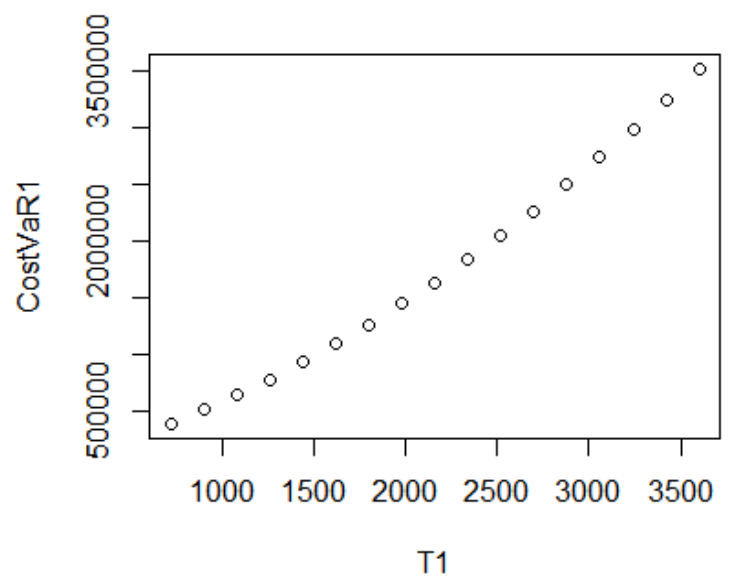

Fig. 5. Right tail $5 \%$ VaR of warranty cost (on the $Y$-axis) against $T_{1}$ (on the $X$-axis).

The feasible range of $P_{1}$ under the constraint is $\left[c p_{l}, c p_{u}\right]$, where both $c p_{l}$ and $c p_{u}$ are determined by $\delta$.

If $P_{1}=3,000$ is known and $T_{1}=720+180 v$ (for $v=1,2, \ldots, 16$ ), the relationship between $T_{1}$ and $\mathrm{CVaR}_{0.05}\left(\omega_{1}\right)$ is illustrated in Fig. 8. Then the feasible range of $T_{1}$ under the constraint is $\left[720, c t_{u}\right]$ where $c t_{u}$ is determined by $\delta$.

\subsection{Three product scenario}

Assume 7 types of components are installed in 3 products, each of which is composed of 4 or 5 components, as illustrated in Table 3. The components in each product are structured in series, which implies: if a component in an product item fails, the item fails.

Assume that the claim arrival processes of the components are NHPPs with a cumulative 


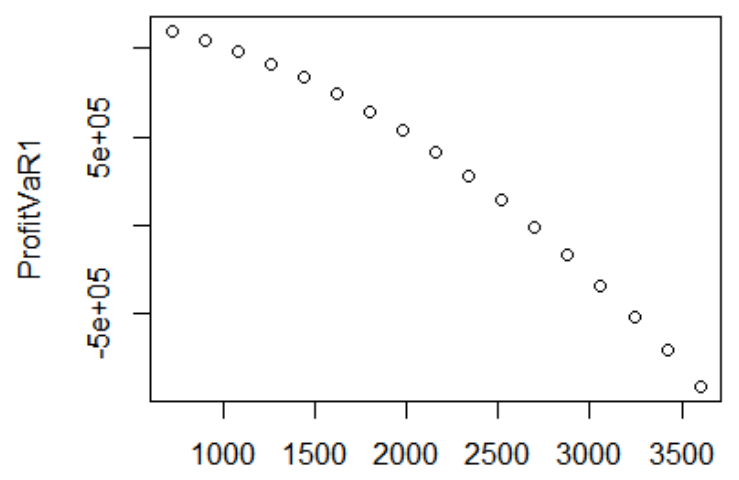

$\mathrm{T} 1$

Fig. 6. $\operatorname{VaR}_{0.05}\left(\omega_{1}\right)$ (on the $Y$-axis) against $T_{1}$ (on the $X$-axis).

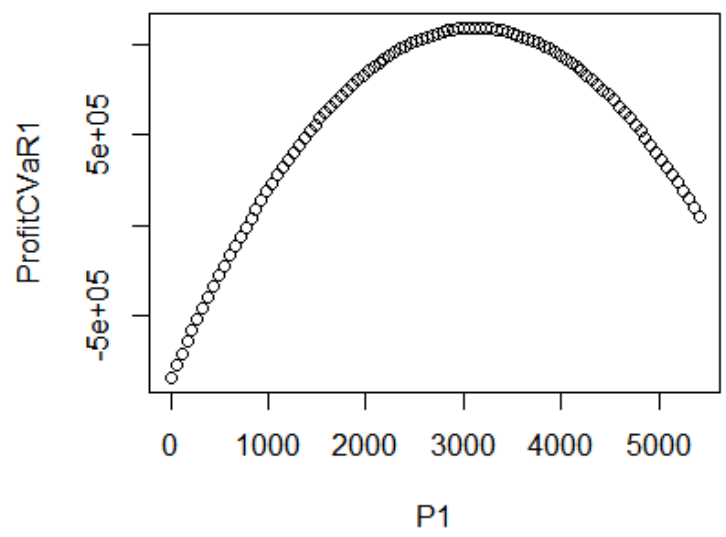

Fig. 7. CVaR1 (on the $Y$-axis) against $P_{1}$ (on the $X$-axis).

Table 3

Components of products

\begin{tabular}{|c|c|c|c|c|c|c|c|}
\hline & \multicolumn{7}{|c|}{ Components } \\
\hline Product & C1 & C2 & C3 & C4 & C5 & C6 & C7 \\
\hline Product1 & $\checkmark$ & $\checkmark$ & & $\checkmark$ & $\checkmark$ & $\checkmark$ & \\
\hline Product2 & $\checkmark$ & & $\checkmark$ & & $\checkmark$ & $\checkmark$ & \\
\hline Product3 & $\checkmark$ & & & $\checkmark$ & & $\checkmark$ & $\checkmark$ \\
\hline
\end{tabular}

failure intensity, $\Lambda(t)=a t^{b}$. The parameters in the claim arrival process models and the warranty claim costs of the components are presented in Table 4 . Then, the claim arrival processes of the products are NHPPs. As such their intensities are given by $\Lambda_{1}(t)=a_{1} t^{b_{1}}+a_{2} t^{b_{2}}+a_{4} t^{b_{4}}+$ $a_{5} t^{b_{5}}+a_{6} t^{b_{6}}, \Lambda_{2}(t)=a_{1} t^{b_{1}}+a_{3} t^{b_{3}}+a_{5} t^{b_{5}}+a_{6} t^{b_{6}}$ and $\Lambda_{3}(t)=a_{1} t^{b_{1}}+a_{4} t^{b_{4}}+a_{6} t^{b_{6}}+a_{7} t^{b_{7}}$, 


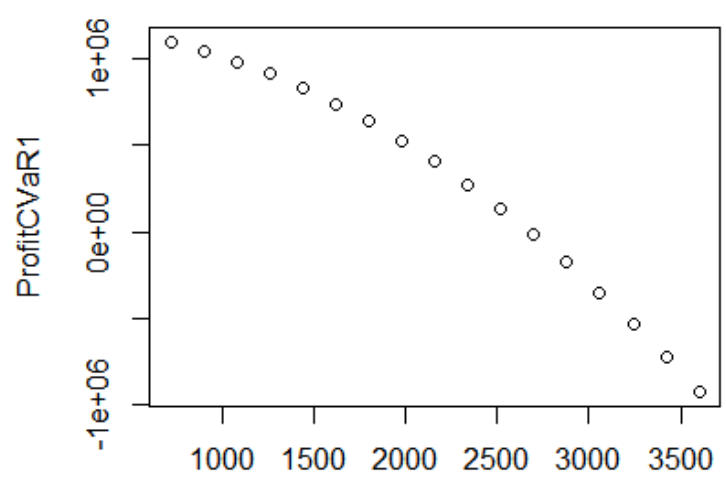

$\mathrm{T} 1$

Fig. 8. $\mathrm{CVaR}_{0.05}\left(\omega_{1}\right)$ (on the $Y$-axis) against $P_{1}$ (on the $X$-axis).

Table 4

Parameters of Components

\begin{tabular}{|l|l|l|l|}
\hline Components & $\mathrm{a}$ & $\mathrm{b}$ & Cost of each claim \\
\hline C1 & 0.0037 & 1.02 & 100 \\
\hline C2 & 0.0037 & 1.01 & 120 \\
\hline C3 & 0.0028 & 1.02 & 80 \\
\hline C4 & 0.0028 & 1.02 & 90 \\
\hline C5 & 0.0024 & 1.03 & 65 \\
\hline C6 & 0.0019 & 1.03 & 60 \\
\hline C7 & 0.0014 & 1.04 & 50 \\
\hline
\end{tabular}

respectively.

According to the above setting, one can generate three data sets, each of which contains claim times and cost of claims of $M_{k}$ items of product $k$. These three data sets are correlated, based on which we can estimate the correlation parameters of our model.

Assume all products have 2-year (720 days) warranty, i.e. $T_{1}=T_{2}=T_{3}=720$. The products' sales volume and warranty cost parameter are then given in Table 5.

The expected warranty cost of the three products are, $\mathrm{E}\left[S_{1}\right]=879.73 \times\left(1,093.6-0.2 P_{1}\right)$, $\mathrm{E}\left[S_{2}\right]=601.65 \times\left(2,079.2-0.5 P_{2}\right)$ and $\mathrm{E}\left[\omega_{3}\right]=572.91 \times\left(2,672-0.6 P_{3}\right)$, respectively. The variances of the warranty costs of the three products are $\operatorname{Var}\left[\omega_{1}\right]=83,376.02 \times\left(1,093.6-0.2 P_{1}\right)$, $\operatorname{Var}\left[\omega_{2}\right]=49,020.67 \times\left(2,079.2-0.5 P_{2}\right)$ and $\operatorname{Var}\left[\omega_{3}\right]=48,238.49 \times\left(2,672-0.6 P_{3}\right)$, respectively. Based on these expected values and variances, the warranty cost distributions, $F_{S_{1}}\left(z_{1}\right), F_{S_{2}}\left(z_{2}\right)$ and $F_{S_{3}}\left(z_{3}\right)$, can be approximated.

To model the dependence among the products, one may construct a trivariate copula. In 
Table 5

Parameters of 3 products

\begin{tabular}{|l|l|l|l|l|l|}
\cline { 2 - 6 } \multicolumn{1}{c|}{} & \multicolumn{2}{|c|}{ Parameters of sales volume } & \multicolumn{2}{l|}{ Parameters of warranty cost } \\
\cline { 2 - 6 } \multicolumn{1}{c|}{} & $A_{k}$ & $\beta_{k}$ & $\eta_{k}$ & $\mu_{k}$ & $\sigma_{k}$ \\
\hline Product 1 & 1,000 & 0.2 & 0.13 & 89.36 & 21.99 \\
\hline Product 2 & 2,000 & 0.5 & 0.11 & 78.22 & 15.95 \\
\hline Product 3 & 2,600 & 0.6 & 0.10 & 79.04 & 20.20 \\
\hline
\end{tabular}

practice, there are many different copula families existing, a suitable copula can be constructed or selected in two steps. In the first step, referring to the physical situation of the products or the features of empirical operating, a proper copula family can be selected. For example, if the dependence is linear, a copula from the elliptical family can be selected; and if a rank correlation is found in the data, a copula from the Archimedean family can be selected. Additionally, the form of marginal distribution, the tail-dependence, etc. all can influence copula selection. In the second step, the goodness-of-fit of the initially selected copulas can be compared by mean squared errors, Akaike Information Criterion (AIC) and Bayesian Information Criterion (BIC). The details of copula selection in warranty data analysis will be investigated in future research.

In this case, considering the non-elliptical marginal distributions and the potential upper tail-dependence, a trivariate Gumbel copula is simply constructed as an example. Then the joint distribution of the number of claims is

$$
\begin{aligned}
H\left(z_{1}, z_{2}, z_{3}\right) & =C\left(u_{1}, u_{2}, u_{3} ; \theta_{1}, \theta_{2}\right) \\
& =\exp \left\{-\left[\left(-\ln u_{1}\right)^{\theta_{1}}+\left[\left(-\ln u_{2}\right)^{\theta_{2}}+\left(-\ln u_{3}\right)^{\theta_{2}}\right]^{\theta_{1}}\right]^{\frac{1}{\theta_{1}}}\right\},
\end{aligned}
$$

where $u_{1}=F_{N_{1}}\left(z_{1}\right), u_{2}=F_{N_{2}}\left(z_{2}\right)$ and $u_{3}=F_{N_{3}}\left(z_{3}\right)$. With simulation, $\theta_{1}=\frac{1}{1-0.59} \approx 2.44$ and $\theta_{2}=\frac{1}{1-0.71}=3.45$.

The density of the joint distribution is given by

$$
h\left(z_{1}, z_{2}, z_{3}\right)=c\left(u_{1}, u_{2}, u_{3}\right) f_{N_{1}}\left(z_{1}\right) f_{N_{2}}\left(z_{2}\right) f_{N_{3}}\left(z_{3}\right)
$$

The cumulative distribution function of the total number of claims of the three products, $\boldsymbol{N}=N_{1}+N_{2}+N_{3}$, of the manufacturer is given by,

$$
F_{\boldsymbol{N}}(z)=\sum_{z_{1}=0, z_{2} \leq z-z_{1}}^{z} h\left(z_{1}, z_{2}, z-z_{1}-z_{2}\right)
$$

The VaR and the CVaR of the total profit $\Omega$ can be determined based on Eqs. (27), (28), and (29). In Table 6 , the $\operatorname{VaR}_{0.05}$ and the $\mathrm{CVaR}_{0.05}$ of the total profit $\Omega$ with different values of $\theta_{2}$ and $\theta_{1}$ are presented.

In Table $6, \theta_{1}$ represents the correlation between the profit of Products 1 and the profit of 
Table 6

The VaR and CVaR of $\Omega$ with different dependences

\begin{tabular}{|l|l|l|l|l|}
\hline \multicolumn{5}{|c|}{$P_{1}=3,173.86, P_{2}=2,380.02, P_{3}=2,513.12$} \\
\hline Copula parameter & $\theta_{1}=1, \theta_{2}=1$ & $\theta_{1}=2.44, \theta_{2}=3.25$ & $\theta_{1}=2.44, \theta_{2}=4$ & $\theta_{1}=3, \theta_{2}=4$ \\
\hline $\operatorname{VaR}_{0.05}(\Omega)$ & $4,884,922$ & $4,859,833$ & $4,859,018$ & $4,858,978$ \\
\hline $\mathrm{CVaR}_{0.05}(\Omega)$ & $4,873,141$ & $4,855,315$ & $4,854,950$ & $4,853,788$ \\
\hline
\end{tabular}

Products 2 and 3 ; and $\theta_{2}$ represents the correlation between the profits of Products 2 and 3 . In the second column, $\theta_{1}=\theta_{2}=1$ implies that the profits of the three products are not correlated; and in the following 3 columns, larger values of $\theta_{1}$ and $\theta_{2}$ indicate a stronger correlations. The $\mathrm{VaR}_{0.05}$ and the $\mathrm{CVaR}_{0.05}$ of the total profit $\Omega$ in the dependent scenarios (Column 3, 4 and 5) are larger than that in the independent scenario (Column 2); and in the dependent scenarios these two values increase with the correlations among the profits of products being stronger. This result implies: if a manufacturer ignores the dependence among warranty claims of products, it will underestimate the upcoming total warranty cost. Such ignorance may cause bias in decision making.

\section{Conclusions}

In the real world, a manufacturer normally produces many different products that have common components installed. Consequently, the frequencies of warranty claims of different products are statistically dependent, which conflicts the fact that the existing methods in the literature solely focus on individual products and ignore the claim dependence.

This paper proposes a method to collectively optimise warranty policy for a portfolio of different products. Using the value-at-rick theory, it attempts to maximise the total profit of a set of products through optimising the warranty price and the warranty length. The numerical example shows that the dependence problem can be properly addressed with the proposed method.

The paper only investigates the optimisation of warranty policy for a portfolio of products covered by a one-dimensional warranty policy. That is, the warranty only covers one dimension, which can be either the usage or the age dimension, but not both. For some products (see Ye and Murthy (2016), for example), however, a warranty policy may cover both age and usage (e.g., the warranty of a car may cover both age and mileage), which is called a two-dimensional warranty policy. Our future work aims to investigate the optimisation problem for products with a two-dimensional warranty coverage. 


\section{Acknowledgement}

The second author acknowledges support from grant number ES/L011859/1, from The Business and Local Government Data Research Centre, funded by the Economic and Social Research Council to provide researchers and analysts with secure data services.

\section{References}

Adkins, R., \& Paxson, D. (2017). Replacement decisions with multiple stochastic values and depreciation. European Journal of Operational Research, 257(1), 174-184.

Aggrawal, D., Anand, A., Singh, O., \& Singh, J. (2014). Profit maximization by virtue of price and warranty length optimization. Journal of High Technology Management Research, $25(1), 1-8$.

Ahmadi, R. (2016). An optimal replacement policy for complex multi-component systems. International Journal of Production Research, 54(17), 5303-5316. doi: 10.1080/00207543.2016.1173252

Artzner, P., Delbaen, F., Eber, J.-M., \& Heath, D. (1999). Coherent measures of risk. Mathematical Finance, $9(3)$, 203-228.

Babaei, S., Sepehri, M., \& Babaei, E. (2015). Multi-objective portfolio optimization considering the dependence structure of asset returns. European Journal of Operational Research, $244(2), 525-539$.

Bai, J., \& Pham, H. (2006). Cost analysis on renewable full-service warranties for multicomponent systems. European Journal of Operational Research, 168(2 SPEC. ISS.), 492-508.

Bee, M. (2016). Density approximations and var computation for compound poisson-lognormal distributions. Communications in Statistics: Simulation and Computation, 1-17.

Bernard, C., \& Vanduffel, S. (2015). A new approach to assessing model risk in high dimensions. Journal of Banking and Finance, 58, 166-178.

Boubaker, H., \& Sghaier, N. (2013). Portfolio optimization in the presence of dependent financial returns with long memory: A copula based approach. Journal of Banking and Finance, 37(2), 361-377.

Chen, C.-K., Lo, C.-C., \& Weng, T.-C. (2017). Optimal production run length and warranty period for an imperfect production system under selling price dependent on warranty period. European Journal of Operational Research, 259(2), 401-412.

Huang, H.-Z., Liu, Z.-J., \& Murthy, D. (2007). Optimal reliability, warranty and price for new products. IIE Transactions, 39(8), 819-827.

Ladany, S., \& Shore, H. (2007). Profit maximizing warranty period with sales expressed by a demand function. Quality and Reliability Engineering International, 23(3), 291-301. 
Lei, Y., Liu, Q., \& Shum, S. (2017). Warranty pricing with consumer learning. European Journal of Operational Research, 263(2), 596-610.

Lin, P.-C., Wang, J., \& Chin, S.-S. (2009). Dynamic optimisation of price, warranty length and production rate. International Journal of Systems Science, 40(4), 411-420.

Liu, B., Wu, J., \& Xie, M. (2015). Cost analysis for multi-component system with failure interaction under renewing free-replacement warranty. European Journal of Operational Research, 243(3), 874-882.

Luo, X., \& Shevchenko, P. V. (2009). Computing tails of compound distributions using direct numerical integration. Journal of Computational Finance, 13(2), 73-111.

Matis, T. I., Jayaraman, R., \& Rangan, A. (2008). Optimal price and pro rata decisions for combined warranty policies with different repair options. IIE Transactions, 40(10), 984-991.

Sklar, A. (1959). Fonctions de répartition àn dimensions et leurs marges. Publications de l'Institut de Statistique de l'Université de Paris, 8, 229-231.

WarrantyWeek. (2016). Thirteenth annual product warranty report.

Wei, J., Zhao, J., \& Li, Y. (2015). Price and warranty period decisions for complementary products with horizontal firms' cooperation/noncooperation strategies. Journal of Cleaner Production, 105, 86-102.

Wu, C.-C., Chou, C.-Y., \& Huang, C. (2009). Optimal price, warranty length and production rate for free replacement policy in the static demand market. Omega, 37(1), 29-39.

Wu, S. (2014a). Construction of asymmetric copulas and its application in two-dimensional reliability modelling. European Journal of Operational Research, 238(2), 476-485.

Wu, S. (2014b). Warranty return policies for products with unknown claim causes and their optimisation. International Journal of Production Economics, 156, 52-61.

Xie, W., Liao, H., \& Zhu, X. (2014). Estimation of gross profit for a new durable product considering warranty and post-warranty repairs. IIE Transactions (Institute of Industrial Engineers), 46(2), 87-105.

Yazdian, S., Shahanaghi, K., \& Makui, A. (2016). Joint optimisation of price, warranty and recovery planning in remanufacturing of used products under linear and non-linear demand, return and cost functions. International Journal of Systems Science, 47(5), 1155-1175.

Ye, Z.-S., \& Murthy, D. P. (2016). Warranty menu design for a two-dimensional warranty. Reliability Engineering \& System Safety, 155, 21-29. 


\section{Appendix}

Proof of Proposition 1.

Proof. The Hessian matrix of $\mathrm{E}\left[\omega_{k}\right]$ is

$$
\boldsymbol{H}_{f\left(P_{k}, T_{k}\right)}=\left[\begin{array}{cc}
-2 \beta_{k} & a_{k} b_{k} \beta_{k} \mu_{k} T_{k}^{b_{k}-1}+\eta_{k} \\
a_{k} b_{k} \beta_{k} \mu_{k} T_{k}^{b_{k}-1}+\eta_{k}-a_{k} b_{k} \mu_{k} T_{k}^{b_{k}-2}\left[\left(b_{k}-1\right)\left(A_{k}-\beta_{k} P_{k}+\eta_{k} T_{k}\right)+2 \eta_{k} T_{k}\right]
\end{array}\right],
$$

and the eigenvalues of $\boldsymbol{H}_{\mathrm{E}\left[\omega_{k}\right]}$ are

$$
\begin{aligned}
x_{1}= & -\left\{2 \beta_{k}+a_{k} b_{k} \mu_{k} T^{b-2}\left[\left(b_{k}-1\right) A_{k}-\left(b_{k}-1\right) \beta_{k} P_{k}+\left(b_{k}+1\right) \eta_{k} T_{k}\right]\right\} \\
& +\sqrt{\left\{2 \beta_{k}-a_{k} b_{k} \mu_{k} T^{b-2}\left[\left(b_{k}-1\right) A_{k}-\left(b_{k}-1\right) \beta_{k} P_{k}+\left(b_{k}+1\right) \eta_{k} T_{k}\right]\right\}^{2}+4\left(a_{k} b_{k} \beta_{k} \mu_{k} T^{b-1}+\eta_{k}\right)^{2}}
\end{aligned}
$$

and

$$
\begin{aligned}
x_{2}= & -\left\{2 \beta_{k}+a_{k} b_{k} \mu_{k} T^{b-2}\left[\left(b_{k}-1\right) A_{k}-\left(b_{k}-1\right) \beta_{k} P_{k}+\left(b_{k}+1\right) \eta_{k} T_{k}\right]\right\} \\
& -\sqrt{\left\{2 \beta_{k}-a_{k} b_{k} \mu_{k} T^{b-2}\left[\left(b_{k}-1\right) A_{k}-\left(b_{k}-1\right) \beta_{k} P_{k}+\left(b_{k}+1\right) \eta_{k} T_{k}\right]\right\}^{2}+4\left(a_{k} b_{k} \beta_{k} \mu_{k} T^{b-1}+\eta_{k}\right)^{2}}
\end{aligned}
$$

respectively. It can be seen that $x_{1}>0$ and $x_{2}<0$. As such, the Hessian matrix is indefinite. As a result, the global minima of $\mathrm{E}\left[\omega_{k}\right]$ does not exist.

Similarly, the Hessian matrix of $\operatorname{Var}\left[\omega_{k}\right]$ is

$\boldsymbol{H}_{\operatorname{Var}\left[\omega_{k}\right]}=\left[\begin{array}{c}0 \\ -\beta_{k}\left(\sigma_{k}^{2}+\mu_{k}^{2}\right) a_{k} b_{k} T_{k}^{b_{k}-1} \\ -\beta_{k}\left(\sigma_{k}^{2}+\mu_{k}^{2}\right) a_{k} b_{k} T_{k}^{b_{k}-1} a_{k} b_{k}\left(\sigma_{k}^{2}+\mu_{k}^{2}\right)\left[2 \eta_{k} T_{k}^{b_{k}-1}+\left(A_{k}-\beta_{k} P_{k}+\eta_{k} T_{k}\right)\left(b_{k}-1\right) T_{k}^{b_{k}-2}\right]\end{array}\right]$,

and the eigenvalues of $\boldsymbol{H}_{\operatorname{Var}\left[\omega_{k}\right]}$ are $x_{1}=a_{k} b_{k} T_{k}^{b_{k}-1}\left(\sigma_{k}^{2}+\mu_{k}^{2}\right) \eta_{k}+a_{k} b_{k} T_{k}^{b_{k}-1}\left(\sigma_{k}^{2}+\mu_{k}^{2}\right) \sqrt{\eta_{k}^{2}+\beta_{k}^{2}}>0$ and $x_{2}=a_{k} b_{k} T_{k}^{b_{k}-1}\left(\sigma_{k}^{2}+\mu_{k}^{2}\right) \eta_{k}-a_{k} b_{k} T_{k}^{b_{k}-1}\left(\sigma_{k}^{2}+\mu_{k}^{2}\right) \sqrt{\eta_{k}^{2}+\beta_{k}^{2}}<0$, which implies that the Hessian matrix is indefinite and the feasible region of $\left(P_{k}, T_{k}\right)$ defined by the constraint is therefore infinite.

Consequently, the global maxima of the objective function does not exist.

Proof of Proposition 2.

Proof. If $P_{k}$ is known, the first order derivative of the objective function $\mathrm{E}\left[\omega_{k}\right]\left(T_{k}\right)$ is given by

$$
\frac{d \mathrm{E}\left[\omega_{k}\right]\left(T_{k}\right)}{d T_{k}}=-\eta_{k} \mu_{k} a_{k}\left(b_{k}+1\right) T_{k}^{b_{k}}-\left(A_{k}-\beta_{k} P_{k}\right) \mu_{k} a_{k} b_{k} T_{k}^{b_{k}-1}+\eta_{k} P_{k}
$$


and the second order derivative of $\mathrm{E}\left[\omega_{k}\right]\left(T_{k}\right)$ is given by

$$
\frac{d^{2} \mathrm{E}\left[\omega_{k}\right]\left(T_{k}\right)}{d T_{k}^{2}}=-a_{k} b_{k} \mu_{k} T_{k}^{b_{k}-2}\left[\left(b_{k}-1\right)\left(A_{k}-\beta_{k} P_{k}+\eta_{k} T_{k}\right)+2 \eta_{k} T_{k}\right]<0,
$$

as a result, the function $\mathrm{E}\left[\omega_{k}\right]\left(T_{k}\right)$ is concave for $T_{k} \geq 0$.

If $P_{k}$ is known, the first order derivative of $\operatorname{Var}\left[\omega_{k}\right]\left(T_{k}\right)$ is

$$
\frac{d \operatorname{Var}\left[\omega_{k}\right]\left(T_{k}\right)}{d T_{k}}=a_{k}\left(\sigma_{k}^{2}+\mu_{k}^{2}\right)\left[\eta_{k} T_{k}^{b_{k}}+\left(A_{k}-\beta_{k} P_{k}+\eta_{k} T_{k}\right) b_{k} T_{k}^{b_{k}-1}\right]>0,
$$

and the second order derivative of $\operatorname{Var}\left[\omega_{k}\right]\left(T_{k}\right)$ is

$$
\frac{d^{2} \operatorname{Var}\left[\omega_{k}\right]\left(T_{k}\right)}{d T_{k}^{2}}=a_{k} b_{k}\left(\sigma_{k}^{2}+\mu_{k}^{2}\right)\left[2 \eta_{k} T_{k}^{b_{k}-1}+\left(A_{k}-\beta_{k} P_{k}+\eta_{k} T_{k}\right)\left(b_{k}-1\right) T_{k}^{b_{k}-2}\right]>0
$$

which implies that the function $\operatorname{Var}\left[\omega_{k}\right]\left(T_{k}\right)$ is convex and monotonously increases for $T_{k} \geq 0$ and that the feasible range of $T_{k}$ defined by $\operatorname{Var}\left[\omega_{k}\right]\left(T_{k}\right) \leq \psi$ is finite. Hence, when $P_{k}$ is known. As a result, the solution of the optimisation problem exists.

If $T_{k}$ is known, the first order derivative of the objective function $\mathrm{E}\left[\omega_{k}\right]\left(P_{k}\right)$ is given by

$$
\frac{d \mathrm{E}\left[\omega_{k}\right]\left(P_{k}\right)}{d P_{k}}=A_{k}-2 \beta_{k} P_{k}+\beta_{k} \mu_{k} a_{k} T_{k}^{b_{k}}+\eta_{k} T_{k}
$$

and the second order derivative of the objective function $\mathrm{E}\left[\omega_{k}\right]\left(P_{k}\right)$ is given by

$$
\frac{d^{2} \mathrm{E}\left[\omega_{k}\right]\left(P_{k}\right)}{d P_{k}^{2}}=-2 \beta_{k}<0
$$

The fact that the second order derivative is negative implies: the objective function $\mathrm{E}\left[\omega_{k}\right]\left(P_{k}\right)$ achieves the global maxima at $P_{k}=\frac{A_{k}+\beta_{k} \mu_{k} a_{k} T_{k}^{b k}+\eta_{k} T_{k}}{2 \beta_{k}}$. If $T_{k}$ is known, the constraint is $P_{k} \geq$ $\frac{A_{k}+\eta_{k} T_{k}-\frac{\psi}{a_{k} T_{k}^{b}\left(\sigma_{k}^{2}+\mu_{k}^{2}\right)}}{\beta_{k}}$. Hence, the optimal solution exists if $T_{k}$ is known.

Proposition 3, 4, 5, 6 and 7 can be established based on Propositions 1 and 2 . 